\title{
IMPACTO DE UM MECANISMO DE DISPARO DA CONVECÇÃO NA PRECIPITAÇÃO SIMULADA COM O MODELO REGIONAL BRAMS SOBRE A BACIA AMAZÔNICA DURANTE A ESTAÇÃO CHUVOSA DE 1999
}

\author{
CLÁUDIO MOISÉS SANTOS E SILVA ${ }^{1}$, SAULO RIBEIRO DE FREITAS ${ }^{2}$ \\ ${ }^{1}$ Universidade Federal do Rio Grande do Norte, Centro de Ciências Exatas e da Terra, Departamento de \\ Ciências Atmosféricas e Climáticas, Natal, RN, Brasil \\ ${ }^{2}$ Instituto Nacional de Pesquisas Espaciais, Centro de Previsão de Tempo e Estudos Climáticos, \\ Cachoeira Paulista, SP, Brasil
}

claudiomoises@ccet.ufrn.br, saulo.freitas@cptec.inpe.br

Recebido Março de 2014 - Aceito Setembro de 2014

\begin{abstract}
RESUMO
O objetivo deste artigo é mostrar o impacto da implementação de uma função de disparo da convecção na parametrização convectiva de Grell e Devenyi, contida no modelo atmosférico regional BRAMS (Brazilian developments on Regional Atmospheric Model). Para verificar o ciclo de precipitação foi usado um conjunto de dados do período chuvoso de 1999 no âmbito do projeto Large scale BiosphereAtmosphere Experiment in Amazonia (LBA). A nova função de disparo de convecção é acoplada aos fluxos de calor sensível e latente à superfície. Além disso, possui a vantagem de considerar a média dos primeiros $60 \mathrm{hPa}$ da troposfera para caracterizar a ascenção pseudo adiabática da parcela. Assim, a parcela em ascenção apresenta redução da Convective Available Potential Energy (CAPE), o modelo acumula menos CAPE nas primeiras horas do dia e com isso, o máximo de precipitação é atrasado (embora dependa da região analisada). Um aspecto negativo foi que a precipitação sobre a Cordilheira dos Andes aumentou relativamente à versão original. Conclui-se que o esquema apresenta vantagens e desvantagens e que faz-se necessário avaliar outras parametrizações do modelo, principalmente a troca de energia e massa na interface solo-vegetação-atmosfera a fim de se obter resultados mais realísticos da precipitação sobe a Amazônia.
\end{abstract}

Palavras chave: parametrização, ciclo diário, CAPE.

\begin{abstract}
IMPACT OF A CONVECTIVE TRIGGER FUNCTION ON THE SIMULATED PRECIPITATION BY THE BRAMS REGIONAL MODEL OVER THE AMAZON BASIN DURING THE 1999 RAINY SEASON

The purpose of the present paper is to show the impact of implementing a convecitve trigger function in the Grell and Devenyi cumulus parameterization on the BRAMS (Brazilian developments on Regional Atmospheric Model) simulations. A dataset colected during the 1999 rainy season in the Large Scale Biosphere-Atmosphere Experiment in Amazonia (LBA) project was used to evaluate the diurnal cycle of observed precipitation. The new convective trigger function is coupled to the surface sensible and latent heat fluxes. In addition, the method has the advantage of considering the average of the first $60 \mathrm{hPa}$ lowest tropospheric levels to characterize the ascending pseudo adiabatically parcel. Thus, the Convective Available Potential Energy (CAPE) values are reduced delaying the maximum rainfall (although it is region dependent). A negative aspect is that precipitation is overestimation over the Andes relativelly to the original version model. It is concluded that the methodology presents advantages and disadvantages, thus evaluating other parameterizations is required, specially the soil-vegetation-atmosphere mass and energy exchabges in order to obtain more realistic simulated precipitation values over the Amazon Basin.
\end{abstract}

Keywords: parameterization, diurnal cycle, CAPE. 


\section{INTRODUÇÃO}

Nuvens, principalmente cumulus, desempenham um papel importante no sistema climático. Elas refletem, absorvem e emitem radiação, aquecem a atmosfera através da liberação e redistribuição de calor latente e calor sensível, modulam os processos hidrológicos através da precipitação, alteram, via correntes subsidentes, os processos físicos no interior da Camada Limite Atmosférica (CLA) (Arakawa, 2004). Além disso, atuam no transporte de poluentes atmosféricos como gases e aerossóis da CLA para a Atmosfera Livre (AL), impactando a qualidade do ar (Freitas et al., 2009).

Em modelos numéricos de tempo e clima (global ou regional) as nuvens cumulus são, em geral, determinadas a partir de modelos conhecidos como Parametrização de Nuvens Cumulus (PNC). A primeira PNC foi desenvolvida por Ooyama (1964) e por Charney e Eliassen (1964), que propuseram um mecanismo de cooperação mútua entre a convecção cumulus e o ambiente de grande escala, a Instabilidade Condicional de Segunda Ordem (Conditional Instability of the Second KindCISK). O CISK foi originalmente desenvolvido para simular a dinâmica de ciclones tropicais. Nesse modelo, as nuvens cumulus são responsáveis por gerar energia para os ciclones através da liberação de calor latente de condensação do conteúdo de vapor d'água. Por outro lado, o ciclone fornece umidade via convergência de massa em baixos níveis para alimentar as nuvens.

A ideia de cooperação entre escalas introduzida no CISK foi fundamental para o desenvolvimento e o entendimento de futuras PNC. A partir desse conceito, passou-se a diferenciar uma PNC pelo tipo de cooperação entre as escalas, que é chamado de controle dinâmico ou fechamento da parametrização. Assim, três principais tipos de fechamentos foram desenvolvidos: (i) a intensidade da convecção cumulus como sendo proporcional à integral da convergência vertical de massa (Kuo, 1965); (ii) fechamento baseado em ajuste convectivo (Betts e Miller, 1986); (iii) fluxo de massa (Arakawa e Schubert, 1974 - AS74). Atualmente desenvolvem-se PNC mais robustas baseadas em técnicas de conjunto (Grell e Devenyi, 2002) (GD02) ou super conjuntos (Krishnamurti et al., 1999). Além das super parametrizações, que são os Modelos Explícitos de Nuvens (CRM) (Cloud Resolving Models) embebidos em modelos globais em baixa resolução (Grabowski e Smolarkiewicz, 1999).

Passadas mais de cinco décadas desde os trabalhos de Ooyama (1964) e de Charney e Eliassen (1964), parametrizar cumulus ainda é um desafio para a meteorologia. Os CRM e as técnicas de conjuntos e super conjuntos são amplamente difundidas; contudo, a operacionalização desses métodos ainda não é uma realidade nos centros de previsão de tempo na grande maioria dos países, a exemplo do Brasil. Em geral a dificuldade é o pouco recurso humano especializado na utilização desses modelos e o alto dispêndio computacional que estes demandam.
Sendo aproximações é comum que as PNC falhem em muitas situações. Um exemplo clássico de erro é a simulação do ciclo diário de precipitação. De forma geral, as PNC simulam os máximos de atividade convectiva entre 4 e 6 horas antes da precipitação observada. Betts e Jakob (2002) sugerem que isso pode estar associado à subestimativa do papel dos cumulus rasos nos MCGA. De fato, os cumulus rasos preparam o ambiente para a formação de cumulus profundos e esta transição não é bem representada nos modelos. Por outro lado, o erro na simulação do ciclo diário de precipitação pode estar associado à deficiência nas formulações da Função de Disparo de Convecção (FDC). A FDC é uma sequência hierárquica de decisões que determina a ocorrência espaço-temporal da atividade convectiva de uma PNC (Kain e Fritsch, 1992).

Neste sentido, Jakob e Siebesma (2003) (JS03) propuseram uma FDC baseada em equações diagnósticas para a energia estática úmida, umidade específica e velocidade vertical $(w)$ da CA. O transporte vertical turbulento de calor sensível e de calor latente à superfície causa perturbação no campo de energia estática úmida e umidade específica no nível mais baixo do modelo de nuvem. Com isso, as parcelas adquirem empuxo positivo e entram em convecção. Caso uma parcela de ar adquira empuxo suficiente para alcançar o NCL e, se neste nível, $w$ for positivo, então a PNC é acionada.

Santos e Silva et al. (2012) implementaram a FDC de Jakob e Siebesma (2003) na parametrização de Grell e Devenyi (2002) e realizaram análises a partir de dados coletados com um radar banda S em Rondônia durante o verão de 1999. Contudo, Santos e Silva et al. (2012) não exploraram a potencialidade desta metodologia para outras regiões da bacia Amazônica. Os resultados foram muito particulares para a região daquele estudo. Não é possível, por exemplo, a partir de Santos e Silva et al. (2012) saber se essa metodologia desenvolvida atrasa o máximo da precipitação em outras regiões ou se ocorre o contrário. Assim, o objetivo deste estudo é avaliar o impacto da metodologia desenvolvida por esses autores em outras regiões da bacia Amazônica.

\section{Material e métodos}

O período dos experimentos e de coleta de dados ocorreu na ocasião do projeto Tropical Rainfall Measuring Mission and Large-Scale Biosphere-Atmosphere Experiment in Amazonia (TRMM-LBA; Silva Dias et al., 2002) durante os meses de Janeiro e Fevereiro de 1999. Os detalhes dos dados e das simulações são descritas a seguir.

\subsection{Dados}

Durante o TRMM-LBA, a precipitação foi coletada por diferentes fontes. A primeira foi uma rede composta por 35 pluviômetros; a segunda, a precipitação de um radar banda $\mathrm{S}$ 
e precipitação e, por fim, a precipitação derivada do algoritmo 3B42_V6 (Santos e Silva et al., 2011). Uma série de publicações (Santos e Silva et al., 2009a,b; Santos e Silva et al., 2011; Santos e Silva et al., 2012) mostram comparações entre as diferentes fontes de dados (pluviômetros, radar e algoritmo 3B42_V6). Especificamente em Santos e Silva et al. (2009b), verificou-se que o ciclo diário de precipitação obtido via 3B42_V6 é consistente com os dados observados pelo radar, exceto em casos de eventos extremos. Por esse motivo os dados do 3B42_V6 foram usados para avaliar a precipitação no presente artigo.

A avaliação da precipitação simulada na bacia foi feita de forma subjetiva através da precipitação do 3B42_V6. A primeira parte é feita por áreas selecionadas baseadas no trabalho de Marengo et al. (2001), que identificaram o início e fim da estação chuvosa na Bacia Amazônica a partir de um amplo conjunto de dados de precipitação da Agência Nacional de Energia Elétrica para o período de 1979 a 1996.

$\mathrm{Na}$ segunda parte, fez-se uma avaliação através de diagramas de Hövmöller de médias meridionais. Este tipo de diagrama é usado para verificar propagação de ondas ao longo do tempo e, na presente pesquisa, apresenta-se como uma oportunidade de verificação da variabilidade intrassazonalidade na atividade convectiva. Os limites de cada área, conforme determinado por Marengo et al. (2001) (A1 a A6 e A8), além de duas áreas sobre a cordilheira dos Andes (A7 e A9) e as faixas nas quais foram determinados os diagramas de Hövmöller são apresentados na Tabela 1 e são visualizadas na Figura 1.

\subsection{Modelo BRAMS e experimentos numéricos}

O modelo BRAMS é uma versão adaptada aos trópicos da sexta versão do modelo RAMS (Walko et al., 2000), que por sua vez é um modelo não hidrostático construído para simular desde pequenos turbilhões atmosféricos até movimentos em escala planetária. Detalhes das equações e dos métodos (a)

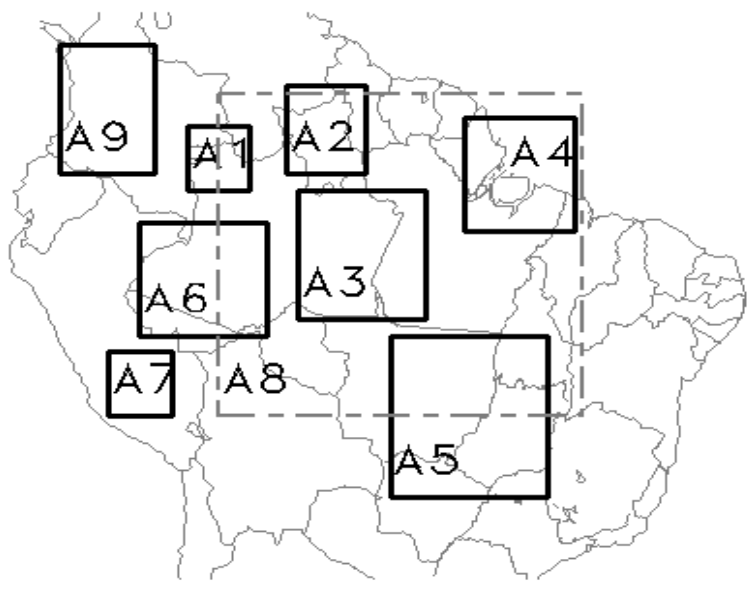

(b)

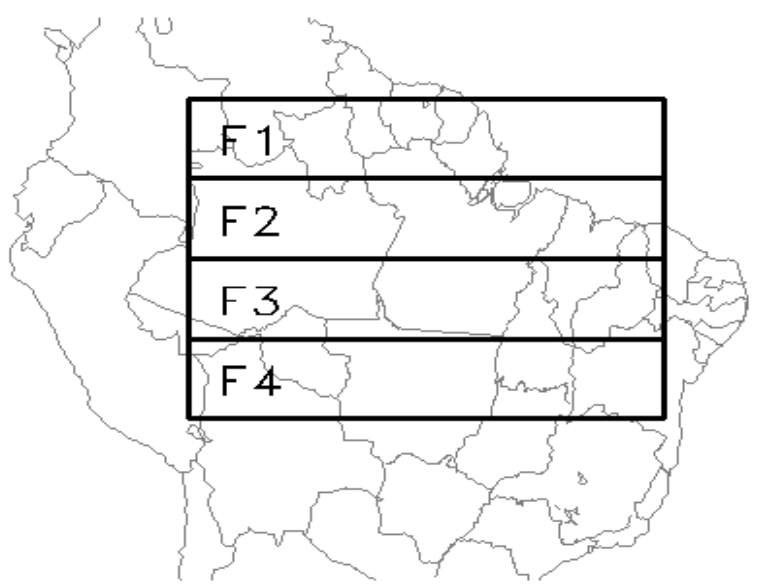

Figura 1 - Área de avaliação de avaliação das simulações realizadas com o modelo regional BRAMS : (a) áreas delimitadas de acordo com Marengo et al. (2001) mais duas sobre os Andes; (b) faixas consideradas para os diagramas de Hövmöller.

Tabela 1 - Experimentos numéricos realizados com o modelo BRAMS.

\begin{tabular}{lcccc}
\hline Experimento & FDC & $\begin{array}{c}\text { Taxa de } \\
\text { entranhamento }\end{array}$ & $\begin{array}{c}\text { Convecção } \\
\text { rasa }\end{array}$ & Fechamento \\
\hline CTRL0 & GD02 & GD02 & desligado & EN1 \\
NENT1 & JS03 & GD02 & desligado & EN1 \\
NENT2 & JS03 & JS03 & desligado & EN1 \\
CTRL1 & GD02 & GD02 & SO99 & EN3 \\
NRAS2 & JS03 & JS03 & SO99 & EN1 \\
NRAS7 & JS03 & GD02 & SO99 & EN2 \\
NRAS8 & JS03 & GD02 & SO99 & EN3 \\
\hline
\end{tabular}


empregados para solucioná-las são encontrados em Freitas (1999).

O modelo BRAMS contém a opção de parametrização de cumulus profundos de Grell e Devenyi (2002) para a convecção profunda. Inicialização diária da umidade do solo (Gevaerd e Freitas, 2006). O BRAMS acoplado ao modelo de transporte de traçadores atmosféricos CATT (Coupled Aerosol and Tracer Transport model) forma o modelo CATT-BRAMS, que é resultado dos trabalhos de Longo (1999) e Freitas (1999) e pode ser visto em detalhes em Freitas et al. (2009).

Como o transporte de traços atmosféricos não faz parte do escopo desta pesquisa utilizou-se a opção de desligar o CATT nas simulações. Assim, somente o modelo atmosférico BRAMS foi empregado nas simulações. Utiliza-se em todos os experimentos a parametrização de convecção profunda de Grell e Devenyi (2002) com fechamento de conjunto. A inicialização da umidade do solo é de Gevaerd e Freitas (2006). A cobertura da vegetação é baseada em Normalized Difference Vegetative Index (NDVI). A parametrização de radiação de ondas longas e ondas curtas é o modelo de Carma. Os coeficientes verticais de difusão turbulenta na CLA de Mellor e Yamada (1974). A microfísica de nuvens tem nível de complexidade 3. Utilizou-se a TSM climatológica. A parametrização da convecção rasa é de Souza (1999). Os experimentos são descritos em Santos e Silva et al. (2012), outros detalhes são apresentados a seguir.

As condições de contorno de grande escala foram provenientes das reanálises do modelo global do ECMWF, com espaçamento de grade de $2,5^{\circ}$, atualizadas a cada 6 horas.
A grade horizontal é centrada na latitude de $5,0 \mathrm{~S}$ e longitude $62,0 \mathrm{~W}$, com espaçamento de grade de $25 \mathrm{~km}$. Acionou-se a parametrização da convecção rasa e da convecção profunda a cada 600 segundos. Foram usados 300 pontos na direção zonal e 200 na meridional. Na vertical fixou-se 40 níveis com espaçamento inicial de $150 \mathrm{~m}$ e razão de incremento de 1,07 até o espaçamento máximo de $950 \mathrm{~m}$, dessa forma o domínio vertical atingiu a profundidade de $22 \mathrm{~km}$.

O solo foi dividido em 7 camadas com profundidades em 0,02 m, 0,06 m, 0,14 m, 0,30 m, 0,62 m, 1,26 m, 2,54 m e $5,10 \mathrm{~m}$. Cada simulação foi iniciada às 00 UTC de 16 de janeiro estendendo-as até 2300 UTC de 28 de fevereiro de 1999. Por possuir um sistema de inicialização de umidade do solo, o modelo BRAMS não necessita de tempo de ajuste (spin up).

Explorou-se quatro características nos experimentos numéricos: (i) o uso da FDC proposta por JS03; (ii) a formulação da taxa de entranhamento de GD02 ou JS03; (iii) os efeitos da parametrização da convecção rasa proposta por Souza (1999) (SO99); (iv) o procedimento de sintonização no fechamento de conjunto da parametrização de GD02. Com isso chegou-se ao total de sete experimentos conforme descritos na Tabela 2.

Foi utilizado o fechamento de conjunto nas simulações; porém, com algumas modificações. Cada conjunto contém 144 membros, composto por três valores de CAPMAX (parâmetro que controla o disparo de convecção original), três eficiências de precipitação e 16 fechamentos. O CAPMAX de referência é $110 \mathrm{hPa}$ com incremento de $20 \mathrm{hPa}$. As três eficiências são definidas em função do cisalhamento do vento. $\mathrm{O}$ controle dinâmico é determinado a partir dos fechamentos GR e KF. O

Tabela 2 - Nomenclatura e delimitação geográfica das áreas de avaliação das simulações.

\begin{tabular}{llcccc}
\hline Área & Regiões e faixas & Latitude inicial & Latitude final & Longitude inicial & Longitude final \\
\hline A1 & Noroeste & $1,0^{\circ} \mathrm{S}$ & $3,0^{\circ} \mathrm{N}$ & $70,0^{\circ} \mathrm{W}$ & $66,0^{\circ} \mathrm{W}$ \\
A2 & Norte & Equador & $5,5^{\circ} \mathrm{N}$ & $63,7^{\circ} \mathrm{W}$ & $58,7^{\circ} \mathrm{W}$ \\
A3 & Central & $9,0^{\circ} \mathrm{S}$ & $1,0^{\circ} \mathrm{S}$ & $63,0^{\circ} \mathrm{W}$ & $55,0^{\circ} \mathrm{W}$ \\
A4 & Litoral & $3,5^{\circ} \mathrm{S}$ & $3,5^{\circ} \mathrm{N}$ & $52,5^{\circ} \mathrm{W}$ & $45,5^{\circ} \mathrm{W}$ \\
A5 & Sudeste & $20,0^{\circ} \mathrm{S}$ & $10,0^{\circ} \mathrm{S}$ & $57,2^{\circ} \mathrm{W}$ & $47,2^{\circ} \mathrm{W}$ \\
A6 & Sudoeste & $10,0^{\circ} \mathrm{S}$ & $3,0^{\circ} \mathrm{S}$ & $73,0^{\circ} \mathrm{W}$ & $65,0^{\circ} \mathrm{W}$ \\
A7 & Andes1 & $15,0^{\circ} \mathrm{S}$ & $11,0^{\circ} \mathrm{S}$ & $75,0^{\circ} \mathrm{W}$ & $71,0^{\circ} \mathrm{W}$ \\
A8 & Bacia & $15,0^{\circ} \mathrm{S}$ & $5,0^{\circ} \mathrm{N}$ & $68,0^{\circ} \mathrm{W}$ & $45,0^{\circ} \mathrm{W}$ \\
A9 & Andes2 & Equador & $8,0^{\circ} \mathrm{N}$ & $78,0^{\circ} \mathrm{W}$ & $72,0^{\circ} \mathrm{W}$ \\
F1 & Faixa1 & Equador & $5,0^{\circ} \mathrm{N}$ & $70,0^{\circ} \mathrm{W}$ & $40,0^{\circ} \mathrm{W}$ \\
$\mathrm{F} 2$ & Faixa2 & $5,0^{\circ} \mathrm{S}$ & $\mathrm{Equador}$ & $70,0^{\circ} \mathrm{W}$ & $40,0^{\circ} \mathrm{W}$ \\
F3 & Faixa3 & $10,0^{\circ} \mathrm{S}$ & $5,0^{\circ} \mathrm{S}$ & $70,0^{\circ} \mathrm{W}$ & $40,0^{\circ} \mathrm{W}$ \\
F4 & Faixa4 & $15,0^{\circ} \mathrm{S}$ & $10,0^{\circ} \mathrm{S}$ & $70,0^{\circ} \mathrm{W}$ & $40,0^{\circ} \mathrm{W}$ \\
\hline
\end{tabular}


fechamento de conjunto EN1 possui 9 membros de GR e 7 de KF. O conjunto EN2 apresenta 12 membros de GR e 4 de KF. Finalmente, EN3 apresenta também 12 membros de GR e 4 de $\mathrm{KF}$, porém aumentando-se em 10 e $20 \%$ o $d t_{c}$.

O experimento de controle (CTRL0) contém a formulação original do disparo de convecção de GD02 e da taxa de entranhamento. No experimento NENTR1 verifica-se o efeito direto da nova FDC com a formulação de entranhamento de GD02, enquanto no experimento NENTR2 utiliza-se o entranhamento de JS03. Nestes três experimentos a parametrização da convecção rasa não é acionada, e o fechamento de conjunto é EN1.

A segunda parte das simulações consiste em acionar a parametrização da convecção rasa de SO99 além da utilização dos fechamentos EN2 e EN3. No experimento CTRL1 verificam-se estes dois efeitos no experimento CTRL0. Em NRAS2 contabilizam-se os efeitos do mecanismo de JS03 associado à parametrização de SO99. Finalmente, os experimentos NRAS7 e NRAS8 contemplam o efeito conjunto da nova FDC implementada a GD02, além da convecção rasa de SO99 e os fechamentos EN2 e EN3, respectivamente.

\section{RESULTADOS}

\subsection{Aspectos gerias das simulações sobre a região do TRMM-LBA}

Na Figura 2 mostra-se o ciclo diário da precipitação simulada com o modelo BRAMS e observada através da rede de pluviômetros e pelo radar S-POL para o período de 19 de janeiro a 28 de fevereiro de 1999. As áreas do S-POL e do BRAMS são descritas em Santos e Silva et al. (2012). A superestimativa da precipitação do dia e subestimativa durante a noite são características comuns a todas as simulações.

O máximo mais intenso simulado ocorre às 14000 UTC $\left(2,0 \mathrm{~mm} \mathrm{~h}^{-1}\right)$ no experimento CTRL0. No experimento CTRL1 o máximo é também às 1400 UTC, porém ligeiramente mais fraco $\left(1,8 \mathrm{~mm} \mathrm{~h}^{-1}\right)$. Após as 1500 UTC, a taxa de precipitação de CTRL1 é maior que em CTRL0, consistente com o resultado de Silva e Souza (2003), que verificam um aumento na precipitação com o uso da parametrização de convecção rasa.

A taxa de entranhamento não acarreta diferenças significativas na precipitação. Entretanto, o máximo de precipitação do experimento NENT1 é uma hora mais tarde que NENT2. Alguns estudos enfatizam a importância do entranhamento na simulação do ciclo diário, por exemplo, Wang et al. (2007) mostram que este causa dois efeitos diretos na simulação do ciclo. O primeiro é a redução da amplitude e o segundo, um atraso no máximo da precipitação em torno de uma hora. A taxa de entranhamento de GD02 é maior que a de JS03. Portanto, consistente com os resultados de Wang et al. (2007).

Quanto ao fechamento, verificam-se implicações importantes na simulação do ciclo diário. Primeiro, suavizam as taxas de precipitação apresentadas na simulação NRAS2 entre 1200 e 1600 UTC. Depois, contribuem para um máximo de precipitação de $1,7 \mathrm{~mm} \mathrm{~h}^{-1}$ às 1800 UTC na simulação NRAS8 e um máximo de $1,5 \mathrm{~mm} \mathrm{~h}^{-1}$ às 1700 UTC em NRAS7.

Dos quatro fatores analisados o uso da FDC de JS03 é o que causa maior impacto no ciclo diário da precipitação

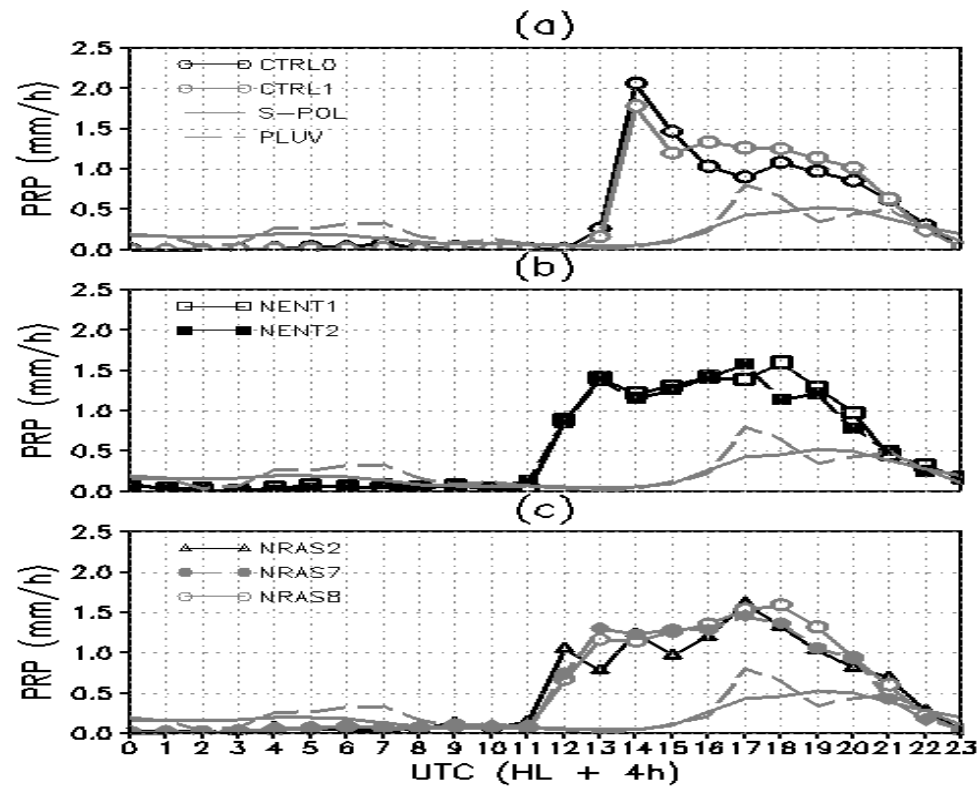

Figura 2 - Ciclo diário da precipitação simulada com o modelo BRAMS e da observada a partir da rede de pluviômetros e com o radar S-POL. 
simulada. A primeira diferença é o disparo da convecção as 1200 UTC, que ocorre duas horas mais cedo que nas simulações realizadas com a FDC de GR02. Entende-se que isso é um impacto negativo conforme verificado pelas curvas das observações. A segunda é a simulação do máximo de precipitação de 3 a 4 horas mais tarde que as verificadas em CTRL0 e CTRL1; considerado, portanto, um impacto positivo. Conforme verificado na Figura 2, a simulação que obteve maior impacto no ciclo diário foi a NRAS8. Com isso, a análise a seguir será entre esta simulação e a simulação CTRL1, que teve pouco impacto quanto ao máximo principal.

\subsection{Avaliação na Bacia Amazônica}

Na Figura 3 mostra-se a precipitação média do período do TRMM-LBA, determinada através do algoritmo 3B42_V6 e simulada através dos experimentos CTRL1 e NRAS8, assim como a diferença entre eles. Em ambos os experimentos a precipitação é elevada sobre a encosta Leste dos Andes, na ZCIT, na costa norte e sobre três Estados nordestinos (Maranhão, Piauí e Ceará). Contudo, no interior da bacia as taxas de precipitação são subestimadas. Considerando a literatura científica do tempo, sabe-se que pelo menos um desses erros é sistematicamente verificado em simulações com outros modelos regionais (Horel et al., 1994; Chou et al., 2005), com simulações individuais usadas em técnicas de superconjuntos (Chaves, et al., 2005), com modelo regional em baixa resolução (Oyama, 2006) ou com um modelo global usando diferentes PNC (Mota e Mendonça, 2006; Pezzi et al., 2008).
Apesar da persistência desses erros, verificam-se melhorias na simulação NRAS8 relativamente ao experimento CTRL1. Essas melhorias são refletidas na diminuição do déficit de precipitação sobre a região da Zona de Convergência do Atlântico Sul (ZCAS), especialmente sobre o Amazonas e Mato Grosso. Outra característica é a diminuição de precipitação sobre o nordeste do Brasil e sobre a encosta Oeste dos Andes. Contudo, sobre a parte Leste das cordilheiras a precipitação aumenta, além de intensificação da Zona de Convergência Intertropical (ZCIT).

O ciclo diário da precipitação simulada nas diversas áreas da bacia e determinado através do algoritmo 3B42_V6 é mostrado na Figura 4. O ciclo observado mostra clara dependência na dimensão espacial, conforme discutido em Santos e Silva et al. (2011) e Santos e Silva (2013). Neste sentido, áreas de pouca extensão, tal como A1 e A2, não apresentam ciclo diário bem definido. Ao contrário, as áreas A3, A5, A6 e A8 exibem ciclos com um máximo bem pronunciado às $2100 \mathrm{UTC}$.

Apesar do máximo na bacia Amazônica ser tipicamente antes das 2100 UTC (Kousky, 1980; Santos e Silva, 2013), o produto 3B42_V6 costuma exibir o máximo mais tardio. Essa diferença é devido ao fato de que este produto usa dados de emissividade de radiação de ondas longas nos topos das nuvens para estimar a precipitação. Então, o máximo de precipitação precede o horário da máxima emissividade dos topos das nuvens, conforme descrito por Huffman et al. (2007).

Na área A4 a curva do 3B42_V6 não indica precipitação mais intensa durante a noite. Contudo, estudos observacionais (a) CTRL1

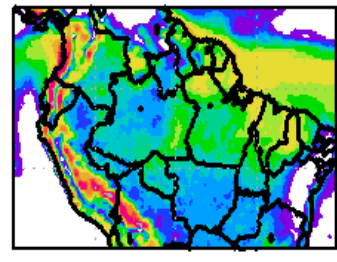

(d) CTRL1-3B42_V6

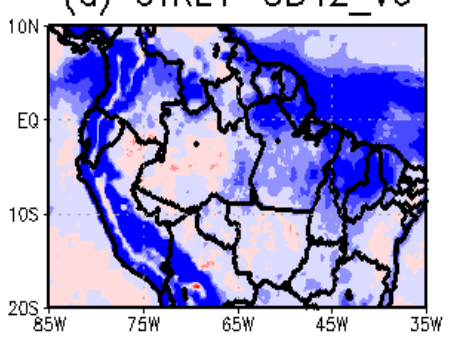

(b) 3B42_V6

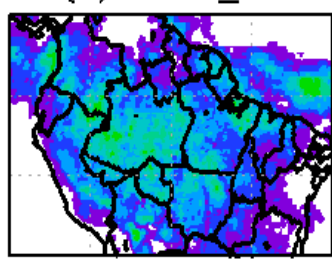

(e) NRAS8-3B42_V6

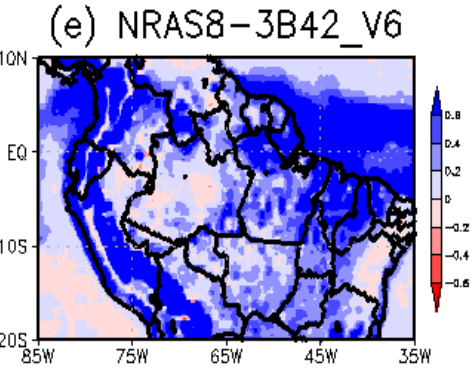

Figura 3 - Precipitação média (mm h-1) para o período de 19 de janeiro a 28 de fevereiro de 1999. Simulada nos experimentos CTRL1 (a) e NRAS8 (c). Estimada através do 3B42_V6 (b). Diferença entre o experimento CTRL1 e 3B42_V6 (d). Diferença entre NRAS8 e 3B42_V6 (e). 


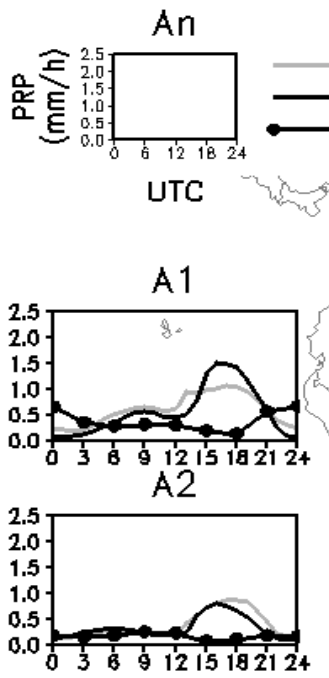

A3

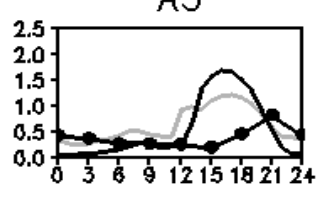

A9
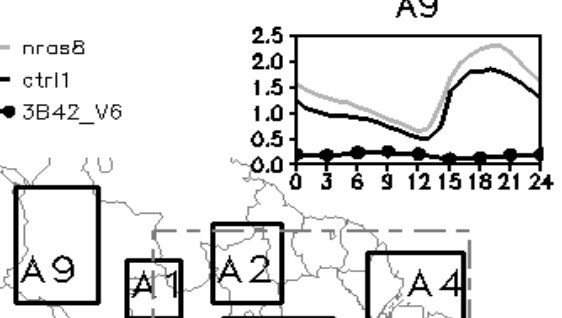
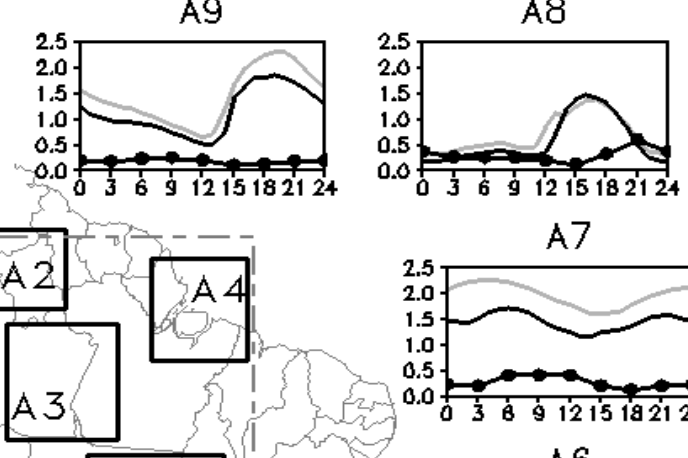

A7

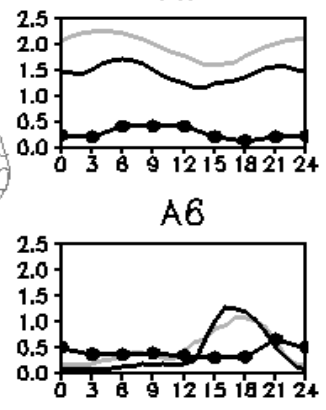

A4

A5
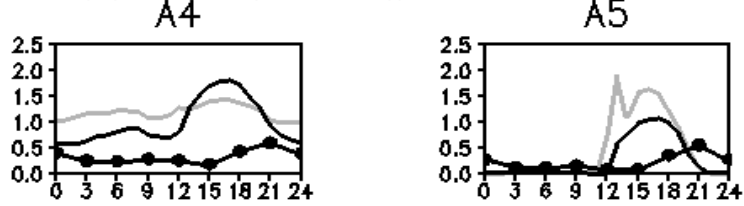

Figura 4 - Ciclo diário da precipitação sobre as áreas selecionadas. No canto superior à esquerda mostra-se a legenda indicando a escala da precipitação no eixo y e o horário em UTC no eixo x; além disso, as linhas indicam os experimentos NRAS8, CTRL1 e a precipitação do 3B42_V6.

indicam que o máximo de atividade convectiva ocorre em torno das 0900 UTC (Kousky, 1980; Negri et al., 2000; Negri et al., 2002; Souza e Rocha, 2006; Santos e Silva, 2013). Neste caso, verifica-se que há uma incerteza associada ao dado do 3B42_V6, que não permite uma comparação direta com as simulações. Entretanto, com base nos artigos citados, pode-se concluir subjetivamente que o experimento NRAS8 apresenta melhorias com relação ao CTRL1. Pois é verificado que o experimento NRAS8 distribui a precipitação de maneira mais uniforme ao longo do dia, inclusive indicando precipitação noturna.

$\mathrm{Na}$ área A3 observa-se que a simulação NRAS8 indica um máximo principal de $1,2 \mathrm{~mm} \mathrm{~h}^{-1}$ às 1800 UTC e um máximo secundário de $0,6 \mathrm{~mm} \mathrm{~h}^{-1}$, às 0700 UTC. Em contrapartida apenas um pico de $1,8 \mathrm{~mm} \mathrm{~h}^{-1}$ às 1700 UTC é verificado na simulação CTRL1. Ou seja, mais atrasado e sem indicar precipitação noturna. Sobre essa área, Negri et al. (2002) mostraram um máximo entre 1800 e 1900 UTC, enquanto Garreaud e Wallace (1997) identificaram um pico secundário de atividade convectiva no início da manhã. Portanto, mais consistente com a simulação NRAS8.

As áreas A1 e A6, que correspondem à parte Oeste da Amazônia, apresentam impacto semelhante no ciclo diário. $\mathrm{O}$ horário da precipitação da tarde no NRAS8 é adiantado em 2 horas relativamente a CTRL1. Além disso, simulou-se uma diminuição da intensidade da precipitação mais consistente com as observações. Da mesma forma que as áreas anteriores, o modelo consegue representar chuva noturna. Nas áreas A2 e A9 a precipitação também atrasa em 2 horas, porém a intensidade do pico é maior.

Finalmente, na área A5, diferentemente das outras regiões, a precipitação do NRAS8 apresenta dois máximos durante o dia, o primeiro de $1,8 \mathrm{~mm} \mathrm{~h}^{-1}$ às 1300 UTC e o segundo de $1,5 \mathrm{~mm} \mathrm{~h}^{-1}$ às 1600 UTC. Neste caso a precipitação simulada no experimento CTRL1, a qual apresenta o máximo de $1,2 \mathrm{~mm} \mathrm{~h}^{-1}$ às $1700 \mathrm{UTC}$, é mais coerente tanto com a curva do 3B42_V6, quanto com os resultados de Negri et al. (2000), que verificam a atividade convectiva mais intensa às $1800 \mathrm{HL}$ sobre o Estado do Mato Grosso. Como foi a única área que o ciclo diário de NRAS8 foi inferior a CTRL1 (relativamente à 3B42_V6), cabem algumas interpretações do resultado, conforme a seguir.

$\mathrm{Na}$ área A5 o disparo da convecção antecipado no experimento NRAS8 é resultado do acúmulo de CAPE (Figura 5) devido ao aumento do fluxo de calor latente à superfície (figura não mostrada). Em JS03, valor elevado de CAPE não é condição suficiente para disparo de convecção, porém necessária, pois o termo de flutuabilidade da equação da energia cinética deve superar o termo de dissipação, para que o ponto de grade seja considerado como convectivo. No estudo observacional de Mendonça e Bonati (2008) mostrou-se que a área A5 é a região de precipitação mais intensa durante eventos de ZCAS. Isso se deve à interação entre a liberação de calor 


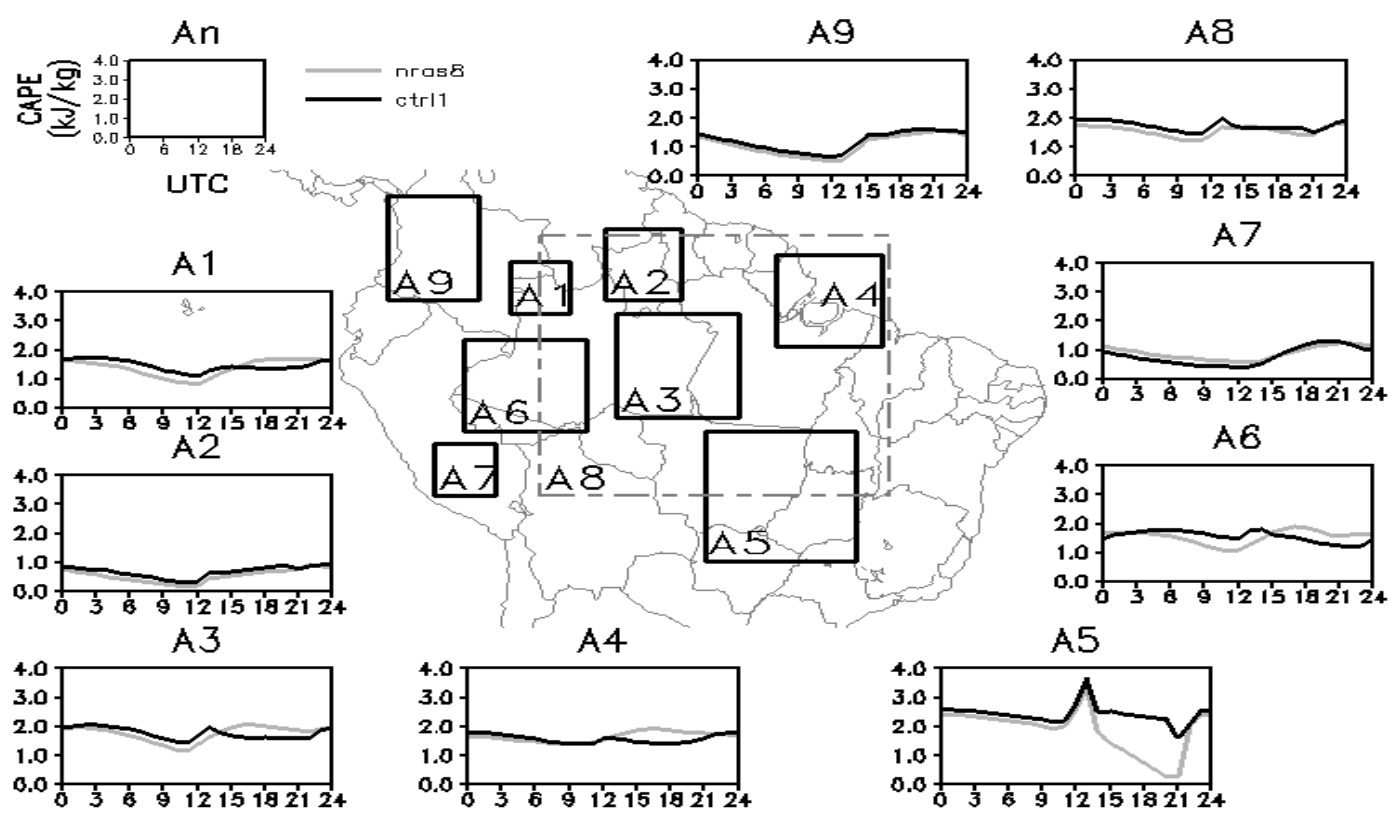

Figura 5 - Idêntica à Figura 4, exceto por não conter a linha correspondente a observação e pela variável ser a CAPE (kJ.kg-1).

latente e os distúrbios de grande escala sobre regiões tropicais (Gandu, 1993). Portanto, o mecanismo de JS03 consegue reproduzir, para o período estudado o papel dos processos de superfície na formação e manutenção de eventos de ZCAS.

Nas outras regiões, há sistematicamente redução da CAPE nas primeiras horas do dia no experimento NRAS8 e acumulo no período após 1500 UTC. Esse acúmulo da CAPE pode ser o mecanismo que explica a formação de precipitação no período noturno, que não se consegue observar no experimento CTRL1. Verificou-se que apenas na área A5 houve redução da CAPE após o pico entre 1200 e 1500 UTC.

Para analisar a bacia Amazônica como um todo faz-se necessário verificar o ciclo diário da área A8. Nele observa-se que a precipitação do NRAS8 é mais intensa por dois motivos: (i) o modelo passa a simular com mais frequência os casos de precipitação noturna; (ii) a precipitação é disparada precocemente entre 1100 e 1300 UTC, estando associada ao acoplamento do mecanismo de disparo aos fluxos de calor à superfície. O máximo de precipitação do experimento CTRL1 é de $1,5 \mathrm{~mm} \mathrm{~h}^{-1}$ às 1600 UTC. Enquanto no experimento NRAS8 é de $1,3 \mathrm{~mm} \mathrm{~h}^{-1}$ às $1700 \mathrm{UTC}$, consistente com os resultados de Bechtold et al. (2004).

Os perfis de aquecimento $\left(Q_{1}\right)$ e secagem $\left(Q_{2}\right)$ da atmosfera devido à convecção profunda são mostrados na Figura 6. As equações para o cálculo de $Q_{1}$ e $Q_{2}$ são deduzidas por Yanai et al. (1973) e descritas a seguir:

$$
Q_{1}=\frac{\partial s}{\partial t}+\overline{\nabla \cdot s V}+\frac{\partial \bar{s} \bar{\omega}}{\partial p}=Q_{R}+L(c-e)-\frac{\partial}{\partial p} \overline{s^{\prime} \omega^{\prime}}
$$

$$
Q_{2}=-L\left(\frac{\partial q}{\partial t}+\overline{\nabla \cdot q V}+\frac{\partial \bar{q} \bar{\omega}}{\partial p}\right)=L(c-e)-L \frac{\partial}{\partial p} \overline{q^{\prime} \omega^{\prime}}
$$

sendo $s$ a energia estática seca da parcela, $V$ o vetor velocidade horizontal, $\omega$ a velocidade vertical em coordenadas isobáricas, $p$ a pressão atmosférica, $Q_{R}$ a taxa de aquecimento radiativo, $L$ o calor latente de condensação, $c$ a taxa de condensação, $e$ a taxa de evaporação e $q$ a umidade específica do ar.

Durante a noite o experimento NRAS8 exibe um núcleo de $Q_{1}$ de $6 \mathrm{~K} \mathrm{dia}^{-1}$ centralizado em $7 \mathrm{~km}$, enquanto o máximo do dia é de $21 \mathrm{~K} \mathrm{dia}^{-1}$ na altura de $6 \mathrm{~km}$. No experimento CTRL1 o aquecimento noturno não ultrapassa $6 \mathrm{~K} \mathrm{dia}^{-1}$. Contudo, durante o dia é da mesma intensidade do experimento NRAS8, porém ocorre às 1600 UTC. O perfil médio de $Q_{l}$ do experimento NRAS8 exibe um máximo na altura de $4,5 \mathrm{~km}$, indicando a maior atividade dos cumulus rasos.

Apresenta-se na Figura 7 o diagrama de Hövmöller de $Q_{1}$ para as F1 e F3 descritas na Figura 2. Apesar da elaboração das figuras para as faixas F2 e F4, os efeitos na simulação foram semelhantes à F1 e F3, respectivamente; portanto, são apresentados apenas os resultados de F1 e F3. Na faixa F1, sobre o oceano (50W a 40W), o experimento NRAS8 exibe maior atividade convectiva durante o intervalo entre $0000 \mathrm{e}$ 0800 UTC, comparado aos horários posteriores.

Apesar do experimento CTRL1 também indicar este padrão, a convecção noturna é mais intensa no experimento NRAS8, o que é mais coerente com estudos prévios, como por exemplo, o de Yang e Smith (2006). A atividade convectiva adentra o continente durante o período noturno. Porém, pela 

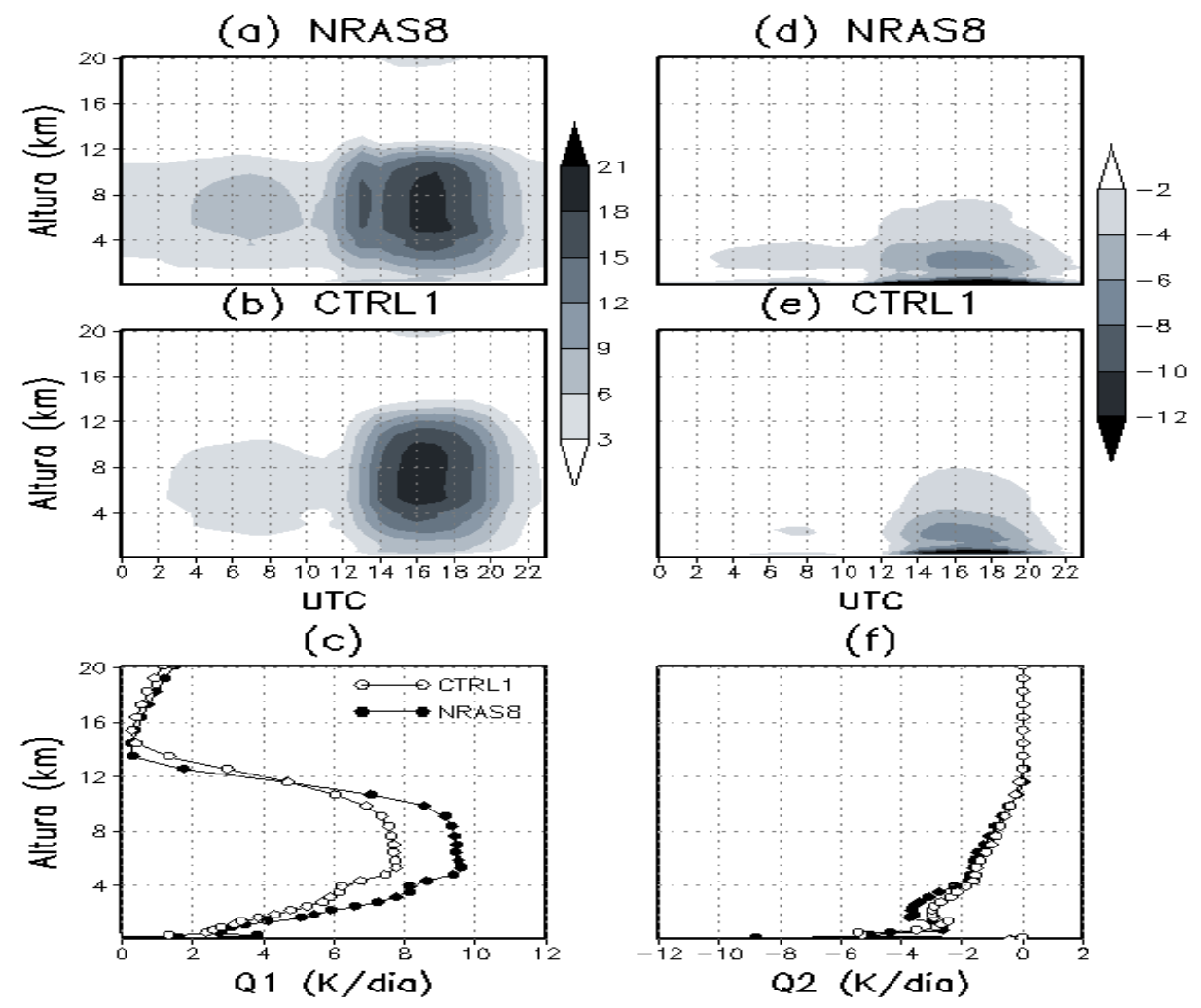

Figura 6 - Ciclo diário e médio do perfil vertical de aquecimento $\left(Q_{1}\right)$ e de secagem $\left(Q_{2}\right)$ para a área A8.

(a) NRAS8

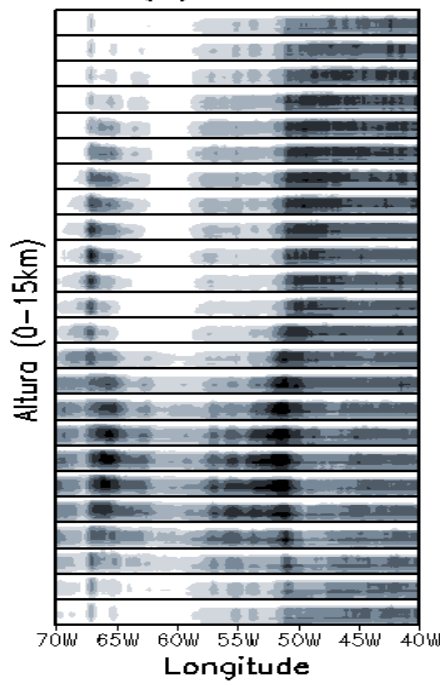

(b) CTRL 1

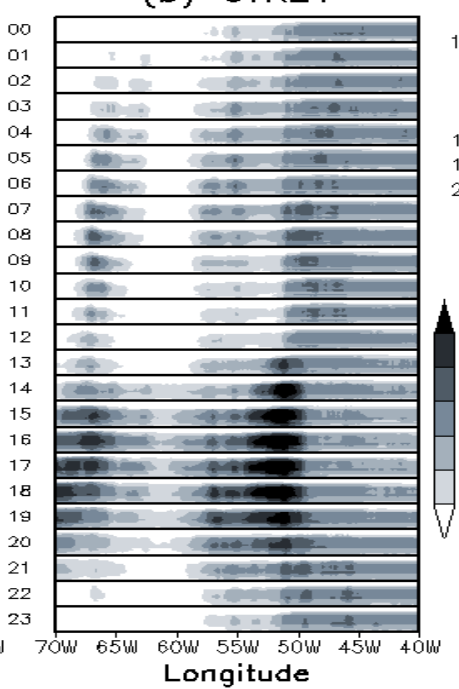

(c)

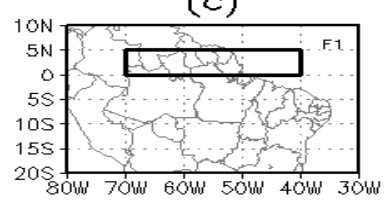

$\because$ NRASB

$\longrightarrow$ CTRL $1^{1}(\mathrm{~d})$

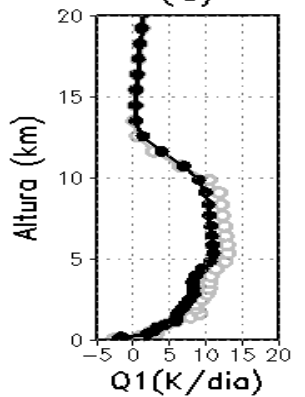

Figura 7 - Diagramas de Hövmöller de $Q_{1}$ para os experimentos NRAS8 (a) e CTRL1 (b); faixa latitudinal dos diagramas destacada pelo retângulo (c); perfil médio de $Q_{1}$ para a área destacada (d).

falta de fontes de calor nestes horários, a convecção é fraca se comparada à do período da tarde (após 1600 UTC). Outra banda convectiva forma-se durante a noite entre $68^{\circ} \mathrm{W}$ e $65^{\circ} \mathrm{W}$ e desloca-se para leste, conforme verificado nos dois experimentos.
Na faixa F3 (Figura 8) a variabilidade intrassazonal é mais evidente e também fica mais clara a vantagem do experimento NRAS8 com relação a CTRL1. Em NRAS8 dois sinais de atividade convectiva noturna associados a diferentes regimes de ventos (Leste e de Oeste, conforme descrito em 
(a) NRAS8

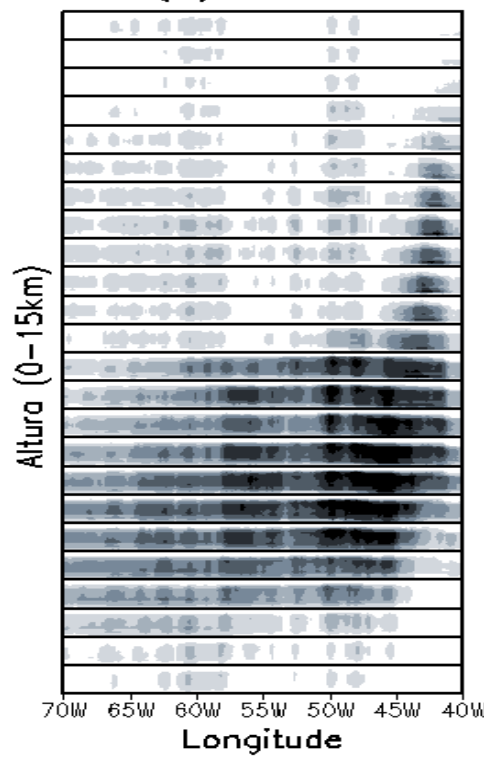

(b) CTRL 1

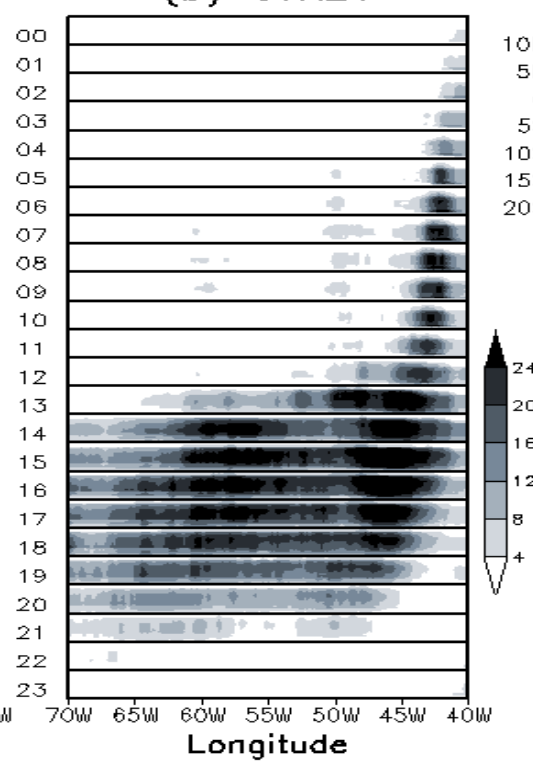

(c)

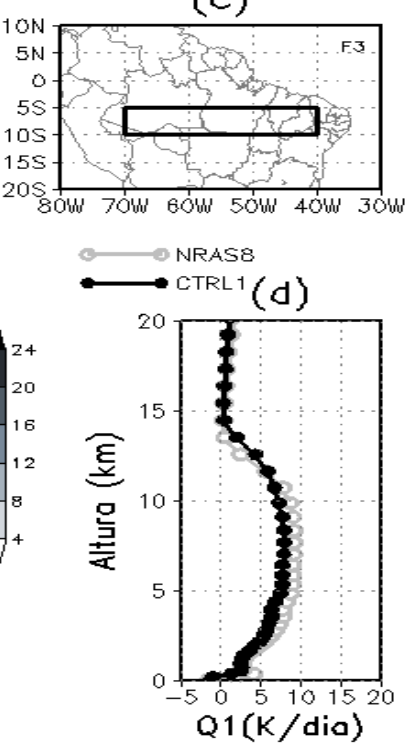

Figura 8 - Idêntica à Figura 7, mas para a faixa F3.

Santos e Silva et al., 2012) são notados, enquanto somente o sinal de Leste é verificado no experimento CTRL1. Outra característica é quanto às taxas de aquecimento mais elevadas no regime de Leste relativamente ao regime de Oeste, verificada no experimento NRAS8, o que é coerente com dados observados (Schumacher et al., 2007) e que não é verificado em CTRL1.

\section{CONCLUSÕES}

Modelos que utilizam parametrização de nuvens cumulus para determinar a precipitação apresentam erros sistemáticos. Dentre estes, destaca-se a representação errônea do ciclo diário, principalmente sobre o continente durante o verão. Para simular o ciclo diário da precipitação de forma coerente, mostra-se que é necessário o acoplamento entre a parametrização de nuvens cumulus e os processos físicos resolvidos ao nível da CLA.

Apesar das melhorias implementadas por Grell e Devenyi (2002) em relação a Grell (1993), o ciclo diário do modelo BRAMS ainda permaneceu com defasagem temporal em relação às observações. Assim Santos e Silva et al. (2012) implementaram a parametrização de Jakob e Siebesma (2003) para o disparo de convecção. Porém, nesse estudo, apenas a região de Rondônia foi analisada. Logo, na presente pesquisa foram apresentados os resultados referentes a diversas regiões da Bacia Amazônica, baseando-se no trabalho de Marengo et al. (2001). A metodologia desenvolvida e os resultados são analisados nos pontos a seguir:

- Uma breve descrição do modelo regional BRAMS é realizada. A grade horizontal do modelo cobre toda a bacia amazônica, tem espaçamento horizontal de 25 $\mathrm{km}$ e domínio vertical de $22 \mathrm{~km}$. As simulações são feitas para o período de 16 de janeiro a 28 de fevereiro de 1999. Os três primeiros dias são excluídos. As condições atmosféricas de grande escala são atualizadas nos contornos laterais a cada 6 horas com as reanálises do modelo global do ECMWF. Ao todo, 7 experimentos são realizados explorando quatro características: taxa de entranhamento, cumulus rasos, fechamento da PNC e mecanismo de disparo de convecção.

- Os resultados das simulações são avaliados através dos dados coletados durante o experimento TRMMLBA. Analisa-se a sensibilidade do modelo a quatro características: (i) implementação de uma nova FDC; (ii) taxa de entranhamento; (iii) uso da parametrização da convecção rasa; (iv) fechamento. A precipitação observada através do radar S-POL exibe um máximo bem pronunciado no final da tarde, típico de regiões continentais durante o verão. De forma geral, todos os experimentos superestimam a precipitação do dia e subestimam (ou não simulam) a precipitação da noite conforme identificado em estudos anteriores (Betts e Jakob, 2002; Colier e Bowman, 2004). Porém, os testes de sensibilidade, especialmente o uso da FDC de JS03, causam impactos positivos na simulação do ciclo diário da precipitação. Assim, detalham-se os resultados de dois experimentos (CTRL1 e NRAS8) que tem apenas a FDC de JS03 por diferença.

- No geral, o máximo de precipitação do experimento 
CTRL1, que é realizado sem acionar a nova FDC ocorre às $1400 \mathrm{UTC}(1000 \mathrm{HL})$. No experimento NRAS8, a precipitação máxima ocorre às 1800 UTC (1400 HL) coerente com o ciclo diário da precipitação observada pelo S-POL (Santos e Silva et al., 2009), com produtos derivados do projeto TRMM (Liu e Zipser, 2008) em com observações de pluviômetros (Marengo et al., 2004; Santos e Silva et al., 2011).

O máximo antecipado de precipitação no experimento CTRL1 deve-se, aparentemente, ao acúmulo de CAPE durante as primeiras horas do dia. Assim, a redução de CAPE no experimento NRAS8, que ocorre em virtude do critério de parcela adotado por JS03, apresenta-se como um mecanismo importante de modulação do ciclo diário conforme observado por Bechtold et al. (2004). É muito provável que esse acúmulo de CAPE se deva aos processos resolvidos pelo modelo de interação Solo-Vegetação-Atmosfera (SVAT) e não necessariamente um problema da parametrização de cumulus em si. Sabe-se que o valor de CAPE é muito sensível à escolha da parcela a ser ascendida adiabaticamente, então superestimativas nos fluxos de calor sensível e calor latente podem provocar aumento de CAPE, conforme discutido por Santos e Silva et al. (2013), então é plausível considerar que além de modificações na parametrização de cumulus, estudos futuros devem atentar-se ao modelo SVAT. Alguns estudos mostram que o ciclo diário de precipitação sobre a parte tropical da América do Sul pode ser bem simulado (por exemplo, Chou et al. 2005; Rocha et al., 2009) mesmo com a parametrização de Grell (1993).

Isso é mais um indício da necessidade de uma boa escolha ou adequação do modelo SVAT para a região. Portanto, como conclusão, considerou-se que a metodologia desenvolvida por Santos e Silva et al. (2012) é satisfatória para seu propósito inicial, que é de simular o máximo mais tarde em relação à simulação controle. Contudo, espera-se que essa metodologia seja potencializada com o uso de um modelo SVAT e que não superestime os fluxos de calor sensível e latente à superfície. Uma proposta é usar o modelo Joint UK Land Environment Simulator (JULES), que foi acoplado recentemente ao sistema CCATT-BRAMS (Moreira et al., 2013).

\section{REFERENCIAS}

ARAKAWA, A. The Cumulus Parameterization Problem: Past, Present, and Future. Journal of Climate, v. 17, n. 13, p. 2493-2525, 2004.

ARAKAWA, A.; SCHUBERT, W.H. Interaction of a cumulus cloud ensemble with the large-scale environment, Part I. Journal of Atmospheric Science, v. 31, n. 3, p. 674-701, 1974.
BECHTOLD, P.; CHABOUREAU, J.P.; BELJAARS, A.; BETTS, A.K.; KÖHLER, M.; MILLER, M.; REDELSPERGER, J.L. The simulation of the diurnal cycle of convection precipitations over land in a global model. Quarterly Journal Royal Meteorological Society, v.130, n. 604, p. 3119-3137, 2004.

BETTS, A.K. Non-precipitating cumulus convection and its parameterization. Quarterly Journal Royal of Meteorological Society, v. 99, n. 419, p. 178-196, 1973.

BETTS, A.K. The scientific basis and objectives of the U.S. subprogram for the GATE. Bulletin of the American Meteorological Society, v. 55, p. 304-313, 1974.

BETTS, A. K.; JAKOB, C. Evaluation of the diurnal cycle of precipitation, surface thermodynamics, and surface fluxes in the ECMWF model using LBA data. Journal of Geophysical Research, v. 107, n. D20, 8045, doi: 10.1029/2002JD000427, 2002.

BETTS, A.K.; MILLER, M.J. A new convective adjustment scheme. Part II: Single column tests using GATE-wave, BOMEX, ATEX, and Arctic Airmass data sets. Quarterly Journal of the Royal Meteorological Society, v. 112, p. 693-710, 1986.

CHARNEY, J.G.; ELIASSEN, A. On the growth of hurricane depression. Journal of the Atmospheric Science, v. 21, n. 1, p. 68-75, 1964.

CHAVES, R.R.; ROSS, R.S.; KRISHNAMURTI, T.N. Weather and seasonal climate prediction for South America using a multi-model superensemble. International Journal of Climatology, v.25, n.14, p. 1881-1914, 2005.

CHOU, S.C.; BUSTAMANTE, J.F.; GOMES, J.L. Evaluation of Eta Model seasonal precipitation forecasts over South America. Nonlinear Processes in Geophysics, v. 12, n. 6, p. 537-555, 2005.

COLLIER, J.C.; BOWMAN, K.P. Diurnal cycle of tropical precipitation in a general circulation model. Journal of Geophysical Research, v. 109, D17105, doi:10.1029/2004JD004818, 2004.

FREITAS, S.R. Modelagem numérica do transporte e das emissões de gases traços e de aerossóis de queimadas no cerrado e floresta tropical. Tese de Doutorado, Instituto de Física, Universidade de São Paulo, 1999.

FREITAS, S.R.; LONGO, K.M.; SILVA DIAS, M.A.F.; CHATFIELD, R.; SILVA DIAS, P.L.; ARTAXO, P.; ANDREAE, M.O.; GRELL, G.; RODRIGUES, L.F.; FAZENDA, A.; PANETTA, J. The Coupled Aerosol and Tracer Transport model to the Brazilian developments on the Regional Atmospheric Modeling System (CATTBRAMS) - Part 1: Model description and evaluation. Atmospheric Chemistry and Physics, v. 9, p. 2843-2861, 2009 . 
GANDU, A.W. Modelagem numérica da resposta troposférica regional à fontes tropicais de calor. Tese (Doutorado em Meteorologia) - Departamento de Ciências Atmosféricas do Instituto Astronômico e Geofísico, Universidade de SãoPaulo, São Paulo, 209 f, 1993.

GARREAUD, R.D.; WALLACE, J.M. The diurnal march of convective cloudiness over Americas. Monthly Weather Review, v. 125, n. 12, p. 3157-3171, dez, 1997.

GEVAERD, R.; FREITAS, S. R. Estimativa operacional da umidade do solo para inicialização de modelos de previsão numérica da atmosfera. Parte I: Descrição da metodologia e validação. Revista Brasileira de Meteorologia, v. 21, n. 3, p. 1-15, 2006.

GRABOWSKI, W.W.; SMOLARKIEWICZ, P.K. CRCP: A cloud resolving convective parameterization for modeling the tropical convective atmosphere. Physica D, v. 133, n. 1-4, p. 171-178, 1999.

GRELL, G. Prognostic evaluation of assumptions used by cumulus parameterization. Monthly Weather Review, v. 121, n. 3, p. 764-787, 1993.

GRELL, G.A.; DEVENYI, D. A generalized approach to parameterizing convection combining ensemble and data assimilation techniques. Geophysical Research Letters, v. 29, n. 14, 1693, doi:10.1029/2002GL015311, 2002.

HOREL, J.D.; PECHMANN, J.B.; HAHMANN, A.N.; GEISLER, J.E. Simulations of the Amazon Basin circulation with a regional model. Journal of Climate, v. 7, n. 1, p. 56-71, 1994.

HUFFMAN, G.J.; ADLER, R.F.; BOLVIN, D.T.; GU, G.; NELKIN, E.J.; BOWMAN, K.; HONG, Y.; STOCKER, E.F.; WOLFF, D.B. The TRMM Multisatellite Precipitation Analysis (TMPA): quasi-global, multiyear, combinedsensor precipitation estimative at fine scales. Journal of Hydrometeorology, v. 8, n. 1, p. 38-55, 2007.

JAKOB, C.; SIEBESMA, A. P. A new subcloud model for massflux convection schemes: influence on triggering, updrafts properties, and model climate. Monthly Weather Review, v. 131, n. 9, p. 2765-2778, 2003.

KAIN, J.S.; FRITSCH, J.M. The role of the convective "trigger function" in numerical forecasts of mesoscale convective systems. Meteorology and Atmospheric Physics, v. 49, n. 1-4, p. 93-106, 1992.

KOUSKY, V.E. Diurnal rainfall variation in northeast Brazil. Monthly Weather Review, v. 108, n. 4, p. 488498, 1980.

KRISHNAMURTI, T.N.; KISHTAWAL, C.M.; LAROW, T.E.; BACHIOCHI, D.R.; ZHANG, Z.; WILLIFORD, C.E.; GADGIL, S.; SURENDRAN, S. Improved weather and seasonal climate forecasts from multimodel superensemble. Science. v. 285, n.5433, p. 1548-1850, 1999.
KUO, H.L. On formation and intensification of tropical cyclones through latent heat release by cumulus convection. Journal of the Atmospheric Sciences, v. 22, n.1, p. 4063, 1965.

LIU, C.; ZIPSER, J. Diurnal cycles of precipitation, clouds, and lightning in the tropics from 9 years of TRMM observations. Geophysical Research Letters, v. 31, L06116, doi: 10.2029/2007GL032437, 2008.

LONGO, K. Estudos de partículas de aerossóis e gases traços na atmosfera da bacia Amazônica: influencias das circulações regionais e de larga escala. Tese de doutorado, Instituto de Física, Universidade de São Paulo, 1999.

MARENGO, J.A.; FISCH, G.; MORALES, C.; VENDRAME, I.; DIAS, P.C. Diurnal variability of rainfall in Southwest Amazonia during the LBATRMM field campaign of the Austral summer of 1999, Acta Amazônica, v. 34, n.4, p. 593-603, 2004.

MARENGO, J.A.; LIEBMANN, B.; KOUSKY, V.; FILIZOLA, N.P.; WAINER, I.C. Onset and end of the rainy season in brazilian Amazon Basin. Journal of Climate, v. 14, n.5, p. 833-852, 2001.

MELLOR, G.L.; YAMADA, T. A hierarchy of turbulence closure models for planetary boundary layers. Journal of the Atmospheric Science, v. 31, n. 7, p. 1791-1806, 1074.

MENDONÇA, R.W.B. E BONATTI, J.P. Estudo da energética modal para episódios de ZCAS. Parte I: análise observacional. Revista Brasileira de Meteorologia, v. 23, n.4, p. 360-380, 2008.

MOREIRA, D.S.; FREITAS, S.R.; BONATTI, J.P.; MERCADO, L.M.; ROSÁRIO, N.M.E.; LONGO, K.M.; MILLER, J.B.; GLOOR, M.; GATTI, L.V. Coupling between the JULES land-surface scheme and the CCATT-BRAS atmospheric chemistry model (JULES-CCATT-BRAMS 1.0): applications to numerical weather forecasting and the $\mathrm{CO} 2$ budget in South America. Geoscientific Model Development, v. 6, p. 1243-1259, 2013.

MOTA, M.A.S. E MENDONÇA, A.M. Comparação dos esquemas de convecção Kuo e RAS usando o modelo atmosférico global do CPTEC durante o experimento WetAMC/LBA. Revista Brasileira de Meteorologia, v. 21, n. 3b, p. 356-370, 2006.

NEGRI, A.J.; ANAGNOSTOU, E.N. ADLER, R.F. A 10-yr climatology of Amazonian rainfall derived from passive microwave satellite observations. Journal of Applied Meteorology, v. 39, n. 1, p. 42-56, 2000.

NEGRI, A.J.; XU L.; ADLER, R.F. A TRMM-calibrated infrared rainfall algorithm, applied over Brazil. Journal of Geophysical Research, V. 107, n. D20, 8048 doi: 10.1029/2000JD000265, 2002. 
OOYAMA, K. A dynamical model for the study of tropical cyclone development. Geophysics International, v. 4, n., p. 187-198, 1964.

OYAMA, M.D. Erros sistemáticos no clima da região tropical da América do Sul simulado pelo modelo regional MM5 em baixa resolução horizontal. Revista Brasileira de Meteorologia, v. 21, n.1, p.118-133, 2006.

PEZZI, L.P.; CAVALCANTI, I.F.A.; MENDONÇA, A.M. A sensitivity study using two different convection schemes over South America. Revista Brasileira de Meteorologia. v. 23, n. 2, p. 170-190, 2008.

ROCHA, R.P.; MORALES, C.A.; CUADRA, S.V.; AMBRIZZI, T. Precipitation diurnal cycle and summer climatology assessment over South America: An evaluation of Regional Climate Model version 3 simulations. Journal of Geophysical Research, v. 114, p. D10108, 2009.

SANTOS E SILVA, C.M. Ciclo diário e semidiário de precipitação na costa norte do Brasil. Revista Brasileira de Meteorologia, v. 28, p. 34-42, 2013.

SANTOS E SILVA, C.M.; FREITAS, S.R.; GIELOW, R. Ciclo diário da precipitação estimada através de um radar banda $\mathrm{S}$ e pelo algoritmo 3B42_V6 do projeto TRMM durante a estação chuvosa de 1999 no Sudoeste da Amazônia. Revista Brasileira de Meteorologia, v. 26, p. 95-108, 2011.

SANTOS E SILVA, C.M.; FREITAS, S.R.; GIELOW, R. Numerical simulation of the diurnal cycle of rainfall in SW Amazon basin during the 1999 rainy season: the role of convective trigger function. Theoretical and Applied Climatology, v. 109, p. 473-483, 2012.

SANTOS E SILVA, C.M.; FREITAS, S.R.; GIELOW, R.; BARROS, S.S. Evaluation of high-resolution precipitation estimate over the Amazon Basin. Atmospheric Science Letters, v. 10, p. 273-278, 2009a.

SANTOS E SILVA C.M.; GIELOW R.; FREITAS S.R. Diurnal and semidiurnal rainfall cycles during the rain season in SW Amazonia, observed via rain gauges and estimated using S-band radar. Atmospheric Science Letters, v.10, n.2, p. 87-93, 2009b.

SCHUMACHER, C.; ZHANG, M.H.; CIESIELSKI, P.E. Heating structure of the TRMM field campaigns. Journal of the Atmospheric Science, v. 64, n. 7, p. 2593-2610, 2007.
SILVA DIAS, M.A.F.; RUTLEDGE, S.; KABAT, P.; SILVA DIAS, P.L.; NOBRE, C.A.; FISCH, G.; DOLMAN, A.J.; ZIPSER, E.; GARSTANG, M.; MANZI, A.O.; FUENTES, J.D.; ROCHA, H.R.; MARENGO, J.; PLANA-FATTORI, A.; SÁ, L.D.A.; ALVALÁ, R.C.S.; ANDREAE, M.O.; ARTAXO, P.; GIELOW, R.; GATTI, L. Cloud and rain processes in a biosphere atmosphere interaction context in Amazon region. Journal of Geophysical Research, v.107, n. D20, 8072, doi: 10.1029/2001JD000335, 2002a.

SILVA, E.M.; SOUZA, E.P. Impacto da implementação de uma parametrização de convecção rasa em um modelo de mesoescala. Descrição e testes de sensibilidade do esquema. Revista Brasileira de Meteorologia, v. 18, n.1, p. 33-42, 2003.

SOUZA, E.P. Estudo teórico e numérico da relação entre convecção e superfícies heterogêneas na região amazônica. 1999, 121p. Tese (Doutorado em Meteorologia) - Instituto de Astronomia, Geofísica e Ciências Atmosféricas, Universidade de São Paulo. São Paulo, 1999.

SOUZA, E.B.; ROCHA, E.P. Diurnal variation of rainfall in Bragança-PA (Eastern Amazon) during rainy season: means characteristics and extreme events. Revista Brasileira de Meteorologia, v. 21, n. 3a, p. 142-152, 2006.

WALKO R.; BAND L.; BARON J.; KITTEL F.; LAMMERS R.; LEE T.; OJIMA D.;PIELKE R.; TAYLOR C.; TAGUE C.; TREMBACK C.; VIDALE P. Coupled atmospherebiophysics-hydrology models for environmental modeling. Journal of Applied Meteorology, v. 39, n. 6, p. 931-944, 2000.

WANG, Y.; ZHOU, L.; HAMILTON, K. Effect of convective entrainment/detrainment on the simulation of the tropical precipitation diurnal cycle. Monthly Weather Review, v. 135, n. 2, p. 567-585, 2007.

YANAI, M.; ESBENSEN, S.; CHU, J.H. Determination of bulk properties of tropical cloud clusters from large-scale heat and moisture budgets. Journal of the Atmospheric Science, v. 30, n. 4, p. 611-627, 1973.

YANG, S.; SMITH, E.A. Mechanism for diurnal variability of global tropical rainfall observed for TRMM. Journal of Climate, v. 19, n. 20, p. 5190-5226, 2006. 